\title{
Climate model under fire as rains fail India
}

\section{K. S. Jayaraman, New Delhi}

As much of southern Asia faces its worst drought for 30 years, the Indian government is being heavily criticized for its reliance on a statistical model to forecast the monsoon.

The India Meteorological Department (IMD), based in New Delhi, uses the model to issue a forecast of the monsoon every spring. This May it issued a prediction saying that rainfall would be $101 \%$ of normal. But by 6 August, this forecast remained way off the mark, with about three-quarters of the country having received very little rain.

Most of India's rain falls during the summer monsoon season between June and September, with July typically accounting for half of the annual total. But this season has been unusually dry, and last week's heavy rain and flooding in some areas are unlikely to save the harvest, most observers say. An accurate forecast of the impending drought would have allowed farmers to switch to more resilient crop varieties, as well as giving the authorities more time to prepare for a potentially poor harvest.

The IMD's statistical model is based on past weather patterns. It tracks 16 factors thought to have some influence on the monsoon, ranging from the amount of snow lying on the Himalayas to the status of the El Niño climate pattern in the eastern Pacific Ocean.

Many researchers point out that the prediction of extreme events - such as the failure of the rains - will always be challenging for this type of model. But some are now

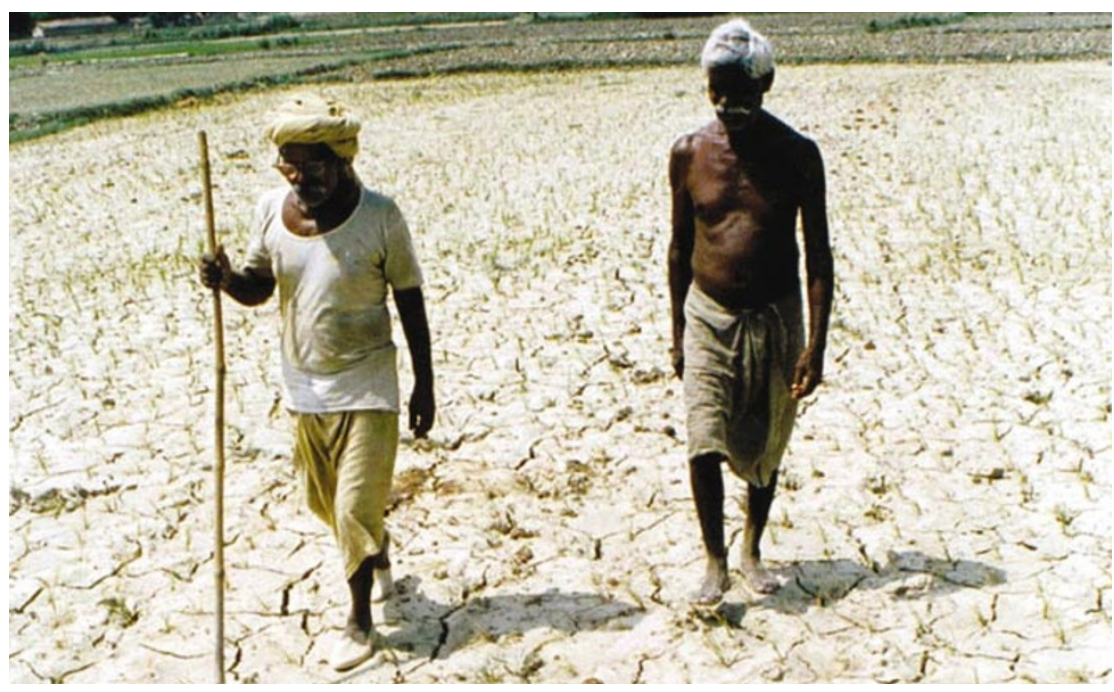

Poor prediction: the Indian government failed to forecast this year's drought, the worst for 30 years.

questioning the IMD's choice of criteria, and others say that the government should switch to more sophisticated generalcirculation models (GCMs). These calculate the evolution of the ocean-atmosphere system by combining dynamic equations with data on initial conditions.

"The real challenge in long-range monsoon forecasting is to predict the extreme drought events," says J. Srinivasan, a climatologist at the Indian Institute of Science in Bangalore. "Because we had normal or above normal rainfall for the past 14 years, we may have placed more faith in statistical evidence, so I try and give answers to the large number of people who are swayable," he said.

Statistics provoked the most heated debate. Some argued that figures bring much-needed perspective; others said that

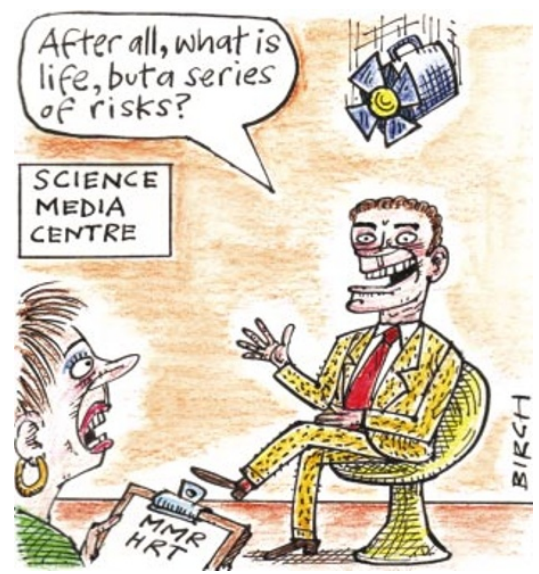

they cloud the issue. For example, media coverage of the suspension of a recent US HRT trial focused on the apparently alarming $26 \%$ increase in the incidence of breast cancer, although this is equivalent to just 8 extra cases per 10,000 women.

Some scientists have already proved to be capable of putting such figures into context. In a 10 July interview on BBC Radio's Today programme, David Purdie, an obstetrician and gynaecologist at the Hull Royal Infirmary, discussed with interviewer John Humphrys the apparently huge increases in risk posed by HRT trials. The figures, he said, are an example of "the Judas factor" - a small number of cases can cause an alarmingsounding percentage if the total number of samples is small. "Christ was betrayed by 8.5\% of his disciples," Purdie told Humphrys. "But when you consider how many actually did the job, it was just one out of 12."

www.sciencemediacentre.org models than we ought to have done."

But the government is playing down the failure of this year's prediction. "It is unfair to blame the model on the basis of one failed forecast in 15 years," says Valangiman Ramamurthy, secretary of the Department of Science and Technology (DST), which oversees the IMD. "We will review the model at the end of the season." He concedes that an effort is already under way to refine the model, however, noting that four of its 16 criteria were changed two years ago.

S. M. Kulshrestha, former director of the IMD, notes that a high degree of variability is intrinsic to the monsoon. "You can throw the model away, but there is nothing to replace it with," he says.

Most researchers see GCMs as the best long-term bet for monsoon prediction, although GCMs cannot yet predict monsoon failure accurately. But they believe that these predictions will improve as more observations are made, the models become more detailed and computers increase in power.

GCMs are already showing some promise. This year, for example, the European Centre for Medium-Range Weather Forecasts model predicted normal Indian rainfall based on conditions on 1 May, but below normal based on 1 June conditions. "This indicates that this year's below normal rainfall occurred on account of changes in atmospheric circulation during May," says Srinivasan.

Officials still doubt whether GCMs can replace the statistical model in the short term. There are lots of GCMs "but no consensus about which one to use", says Dev Raj Sikka, head of the DST's climate-research programme. Nevertheless, Indian scientists agree that this year's unusual monsoon conditions will provide useful data on which to base future predictions. 